\title{
Spatial and molecular mapping of Pfkelch13 gene polymorphism in Africa in the era of emerging Plasmodium falciparum resistance to artemisinin: a systematic review
}

Nadine K Kayiba, Doudou M Yobi, Evariste Tshibangu-Kabamba, Vo P Tuan, Yoshio Yamaoka, Brecht Devleesschauwer, Dieudonné M Mvumbi, Emile Okitolonda Wemakoy, Patrick De Mol, Georges L Mvumbi, Marie-Pierre Hayette, Angel Rosas-Aguirre, Niko Speybroeck

The spread of Plasmodium falciparum isolates carrying mutations in the kelch13 (Pfkelch13) gene associated with artemisinin resistance (PfART-R) in southeast Asia threatens malaria control and elimination efforts. Emergence of PfART-R in Africa would result in a major public health problem. In this systematic review, we investigate the frequency and spatial distribution of Pfkelch13 mutants in Africa, including mutants linked to PfART-R in southeast Asia. Seven databases were searched (PubMed, Embase, Scopus, African Journal Online, African Index Medicus, Bioline, and Web of Science) for relevant articles about polymorphisms of the Pfkelch13 gene in Africa before January, 2019. Following PRISMA guidelines, 53 studies that sequenced the Pfkelch13 gene of 23100 sample isolates in 41 sub-Saharan African countries were included. The Pfkelch13 sequence was highly polymorphic (292 alleles, including 255 in the Pfkelch13-propeller domain) but with mutations occurring at very low relative frequencies. Nonsynonymous mutations were found in only 626 isolates (2.7\%) from west, central, and east Africa. According to WHO, nine different mutations linked to PfART-R in southeast Asia (Phe446Ile, Cys469Tyr, Met476Ile, Arg515Lys, Ser522Cys, Pro553Leu, Val568Gly, Pro574Leu, and Ala675Val) were detected, mainly in east Africa. Several other Pfkelch13 mutations, such as those structurally similar to southeast Asia PfART-R mutations, were also identified, but their relevance for drug resistance is still unknown. This systematic review shows that Africa, thought to not have established PfART-R, reported resistance-related mutants in the past 5 years. Surveillance using PfART-R molecular markers can provide valuable decision-making information to sustain the effectiveness of artemisinin in Africa.

\section{Introduction}

An estimated 219 million new malaria cases and 435000 deaths occurred globally in 2017, with more than $75 \%$ of these cases coming from Africa. ${ }^{1}$ In the absence of an effective vaccine, reducing the burden of Plasmodium falciparum malaria relies on the effectiveness of artemisinin-based combination therapies (ACTs). ${ }^{1,2}$ ACTs combine the rapid antimalarial action (but short halflife) of artemisinin or its derivatives with the slower action (but longer half-life) of partner drugs. ${ }^{3}$ However, one major challenge for malaria control and elimination efforts is the emergence and spread of $P$ falciparum artemisinin resistance (PfART-R) from the Greater Mekong subregion in southeast Asia over the past decade. ${ }^{4-6}$

A reverse migration of PfART-R resistance towards Africa (by comparison with the evolutionary origin and spread of the parasite $)^{7}$ is a troubling scenario that could have severe consequences on the burden of malaria because alternative therapies are few. ${ }^{8-10}$ As this resistance is not yet established in Africa, monitoring PfART-R on the continent is necessary from a global health perspective. ${ }^{9}$ Therapeutic efficacy trials are the standard method for assessing PfART-R; however, insufficient funding restricts these studies in African countries., ${ }^{3,11,12}$ The delayed parasite clearance obtained in clinical trials and the in-vitro ring-stage (trophozoite) survival assay are also useful for tracking the emergence of artemisinin resistance. ${ }^{5,13-15}$
The association of specific single nucleotide polymorphisms (SNPs) in the $P$ falciparum kelch 13 gene (Pfkelch13) with delayed parasite clearance has raised the potential of molecular markers for the surveillance of PfART-R..$^{14,16,17}$ More than 100 Pfkelch13 mutations have been reported in Africa, but there is still little evidence of PfART-R mutants circulating in the continent. ${ }^{9,18}$ In this systematic review, we examine studies reporting Pfkelch13 SNPs across different African countries to determine the relative frequencies and spatial distribution of parasites carrying mutations currently considered to be PfART-R markers.

\section{Methods}

\section{Search strategy and selection criteria}

Our systematic review follows the preferred reporting items for systematic reviews and meta-analyses (PRISMA) guidelines. ${ }^{19,20}$ Seven electronic medical databases (PubMed, Embase, Scopus, African Journal Online, African Index Medicus, Bioline, and Web of Science) were searched for peer-reviewed articles published before January, 2019, that have the relevant population, intervention, comparator, outcomes, and study design (PICOS) framework (appendix p 2).

A predetermined search strategy used French and English versions of keyword terms of the Medical Subject Headings 2018 database and free terms, such as ("malaria" OR "falciparum" OR “paludisme") AND ("marqueur moléculaire" OR "molecular marker" OR
Lancet Infect Dis 2021; 21: e82-92 Published Online October 27, 2020 https://doi.org/10.1016/ S1473-3099(20)30493-X Institute of Health and Society, Université catholique de Louvain, Brussels, Belgium (N K Kayiba MPH,

A Rosas-Aguirre PhD, Prof N Speybroeck PhD); Department of Public Health (N K Kayiba) and Department of Basic Sciences, University of Mbujimayi, Mbujimayi, DR Congo (ETshibangu-Kabamba PhD); Department of Epidemiology and Biostatistics (N K Kayiba, Prof E Okitolonda Wemakoy PhD) and Department of Basic Sciences (DM Yobi MSc, D M Mvumbi PhD, Prof G L Mvumbi PhD), University of Kinshasa, Kinshasa, DR Congo; Department of Endoscopy, Cho Ray Hospital, Ho Chi Minh, Vietnam (V PTuan PhD); Department of Environmental and Preventive Medicine, Oita University, Yufu, Japan (V PTuan, ETshibangu-Kabamba, ProfYYamaoka PhD); Department of Epidemiology and Public Health, Sciensano, Brussels, Belgium (B Devleesschauwer PhD); Department of Veterinary Public Health and Food Safety, Ghent University, Merelbeke, Belgium (B Devleesschauwer); and Department of Parasitology and Mycology, University Hospital of Liège, Liège, Belgium (Prof P De Mol PhD, Prof M-P Hayette PhD)

Correspondence to: Prof Niko Speybroeck, Institute of Health and Society, Faculty of Public Health, Université catholique de Louvain, Brussels 1200, Belgium niko.speybroeck@uclouvain.be See Online for appendix 
For the UNIPROT protein database see https://www. uniprot.org/uniprot/Q8IDQ2 "kelch13" OR "Pfkelch13” OR "K13” OR “PfK13”) AND ("Africa" OR "Afrique" OR each name of the 54 African countries) as detailed in appendix $\mathrm{p} 3$ ). No filters or limitations (ie, language) ensured a large inclusion of informative reports. Individual searches on the internet allowed for the inclusion of references identified in primary reports. Inaccessible full-text articles were requested from corresponding authors. Although previous review papers were not included in the final analysis, they were sources for additional reports. Studies with data from an unknown sampling site or country, systematic reviews, modelling reports, reports based only on genetically modified isolates, conference presentations, abstracts with insufficient information, and letters or correspondence to editors were excluded.

\section{Selection of studies, quality assessment, and data management}

A structured data collection process addressed any possibility of uncertainty or missing data. Two masked teams (NKK, DYM, and AR-A; ET-K, VPT, and YY) worked independently at each stage of the process (literature search, relevance assessment, classification report and validation, and data extraction) before cross-checking and merging related outcomes. Extracted study variables included general information (authors, title of study, year of publication, geographical location of the study), study characteristics (study design, sampling period, collection time during the survey, participant characteristics, setting, sample size, and analytical methods), and SNP information (loci sequenced, alleles found, number of isolates sequenced at specific loci, and number of isolates with individual alleles). The Newcastle-Ottawa scale assessed three quality criteria in selected studies: the representativeness of samples (maximum one star), the sample size (maximum one star), and the study outcome (maximum three stars). ${ }^{21}$ Regarding the outcome, the accurate definition of the genotype (given the reference), and the correct nomenclature of mutations following standard recommendations in molecular diagnostic ${ }^{22,23}$ awarded quality stars to articles. Only moderate (two to three stars) and high (four to five stars) quality studies were included in our systematic review.

\section{Definitions}

PfART-R is defined by a delayed parasite clearance representing partial resistance that affects only ring-stage parasites. ${ }^{17}$ PfART-R mutations are SNPs in the Pfkelch13-propeller domain associated with PfART-R in vitro or in vivo. Our systematic review uses the most updated list of 31 PfART-R molecular markers released by WHO in August, 2018, ${ }^{17}$ to classify PfART-R mutations into three categories. First, validated molecular markers significantly associated with both slow parasite clearance and reduced drug in-vitro sensitivity: Phe446Ile, Asn458Tyr, Met476Ile, Tyr493His, Arg539Thr, Ile543Thr, Pro553Leu, Arg561His, and Cys580Tyr. Second, candidate molecular markers significantly associated with slow parasite clearance: Pro441Leu, Gly449Ala, Cys469Phe, Ala481Val, Pro527His, Asn537Ile, Gly538Val, Val568Gly, Pro574Leu, Phe673Ile, and Ala675Val. Finally, molecular markers suspected to be associated with slow parasite clearance without reaching statistical significance because of the low number of mutants: Asp452Glu, Cys469Tyr, Lys479Ile, Arg515Lys, Ser522Cys, Asn537Asp, Arg575Lys, Met579Ile, Asp584Val, Pro667Thr, and His719Asn.

Mutations similar to molecular markers of PfART-R are also highlighted in this Review because they are structurally close to the WHO PfART-R mutations, although their clinical relevance is not yet established.

\section{Data summary measures and synthesis}

Since studies reporting Pfkelch13 SNPs had different designs and used mainly convenience sampling, their data were not suitable for combining in a meta-analytic approach. Sequencing processes provided variable lengths of genetic sequences resulting in diverse ranges of loci analysed for allele discovery in studies. A narrative synthesis was done to systematically organise the information. SNPs in the Pfkelch13 sequence were defined on the basis of the reference wild-type Pfkelch13 sequence PF3D7_1343700, available in the UNIPROT protein database. Relative frequencies of each allele were summarised using the median values and interquartile ranges. Proportions of mutants with at least one non-synonymous change in the Pfkelch13 gene within each geographical site were calculated. Data from individual reports and locations were geo-referenced and locus-referenced before being uploaded on maps to display spatial and molecular patterns using the ggplot2, ${ }^{24}$ ggmap, ${ }^{25}$ and rgdal ${ }^{26}$ packages in $\mathrm{R}$ (version 3.5 .3 ). ${ }^{27}$ The risk of bias was minimised by excluding studies on malaria cases exported outside Africa and repeated communications on the same isolates.

\section{Results}

A list of 3756 records reporting individual SNPs in the Pfkelch13 gene were identified through database searching, including four records found manually. After removal of 2236 duplicated reports, we screened 1520 records of which 1467 were considered ineligible according to the PICOS approach (figure 1). 53 studies remained after exclusion, with $P$ falciparum sample isolates successfully sequenced for Pfkelch13 in 41 African countries; ${ }^{9,18,28-\pi}$ baseline characteristics of these studies are detailed (appendix pp 4, 7). Analytical methods used in studies were nested PCR with subsequent Sanger sequencing (49 studies), next-generation sequencing of targeted amplicons (two studies), next-generation sequencing of whole $P$ falciparum genomes with a genome-wide association study (one study), and mapping reads to targeted references (one study). Different protocols were used in the exploration of SNPs in the Pfkelch13 gene, with sequence lengths ranging from 445 to 2438 base pairs. 
44 studies identified both Pfkelch13 wild-type and mutant isolates, and nine found only wild-type isolates.

A total of 24652 P falciparum isolates were analysed in the selected studies, yielding 23100 isolates successfully sequenced at loci in the Pfkelch13 gene. The majority of isolates (22474 isolates [97.3\%]) carried a Pfkelch13 gene with either a wild-type sequence or a sequence displaying only synonymous mutations. The remaining 626 isolates $(2 \cdot 7 \%)$ had non-synonymous mutations, $604(2 \cdot 6 \%)$ of which were classified as missense substitutions in the amino acid sequence. Other non-synonymous mutations included 15 insertions $(0.06 \%)$, four deletions $(0.02 \%)$, and three nonsense substitutions ( $0 \cdot 01 \%)$ (appendix p 8$)$.

All the 41 African countries with information about Pfkelch13 polymorphisms were in sub-Saharan Africa (appendix p 9). Pfkelch13 non-synonymous mutations were absent in 11 countries: Botswana, Burundi, GuineaBissau, Liberia, Mauritania, Sierra Leone, Somalia, South Africa, South Sudan, Sudan, and Zimbabwe. Sequence haplotypes were heterogeneous in the other 30 countries, with surveys detecting indistinct wild-type Pfkelch 13 and Pfkelch13 non-synonymous mutations (at least a single isolate carrying a non-synonymous mutation). High $(>50 \%)$ and intermediate $(40-50 \%)$ relative proportions of isolates with non-synonymous Pfkelch13 mutations were reported in west Africa (Senegal and Nigeria) and east Africa (Kenya and Uganda; figure 2).

The majority of Pfkelch13 polymorphic loci (149 of $182[81.9 \%])$ were detected in the propeller domain sequence (ie, downstream of codon position 440), with 255 allelic variations (213 non-synonymous alleles) among a total of 292 alleles found in Pfkelch 13 sequencing studies (figure 3). Lys189Thr was the most frequently reported non-synonymous allele in Pfkelch13 gene sequencing studies and Ala578Ser was the most frequently reported non-synonymous allele in partial sequencing studies of the Pfkelch13-propeller domain. Lys189Thr was observed in 145 mutants with relative frequencies in surveys ranging from $0 \cdot 8 \%$ to $50 \%$ (median $32 \cdot 8 \%$ ), whereas Ala578Ser was reported in 98 mutants at relative frequencies in surveys from $0 \cdot 2 \%$ to $7 \cdot 1 \%$ (median $1 \cdot 4 \%$ ). Lys189Thr and Ala578Ser spanned over several countries at variable relative frequencies and there were no distinctive geographical patterns in their distribution (appendix p 10). Relative frequencies of non-synonymous mutations in the Pfkelch13-propeller domain in surveyed sites ranged from $0 \cdot 08 \%$ to $10 \cdot 3 \%$ (appendix p 8 ).

Only $35(0.15 \%)$ of the total 23100 sequenced isolates recorded in this systematic review had alleles classified as candidate PfART-R markers by WHO: Cys469Tyr (Uganda), ${ }^{29,45}$ Arg515Lys (Zambia), ${ }^{9}$ Ser522Cys (Togo, Central African Republic, Gabon, DR Congo, Uganda, and Kenya), ${ }^{9,42,62,78}$ Val568Gly (Kenya), ${ }^{36}$ Pro574Leu (Rwanda), ${ }^{69}$ and Ala675Val (Uganda and Rwanda). ${ }^{29,45,69}$ Moreover, WHO-validated PfART-R markers were found in at least one isolate in four countries: Phe446Ile (Mali) ${ }^{65}$ Met476Ile (Tanzania), ${ }^{47}$ and Pro553Leu (Kenya and Malawi; figure 4). ${ }^{77}$

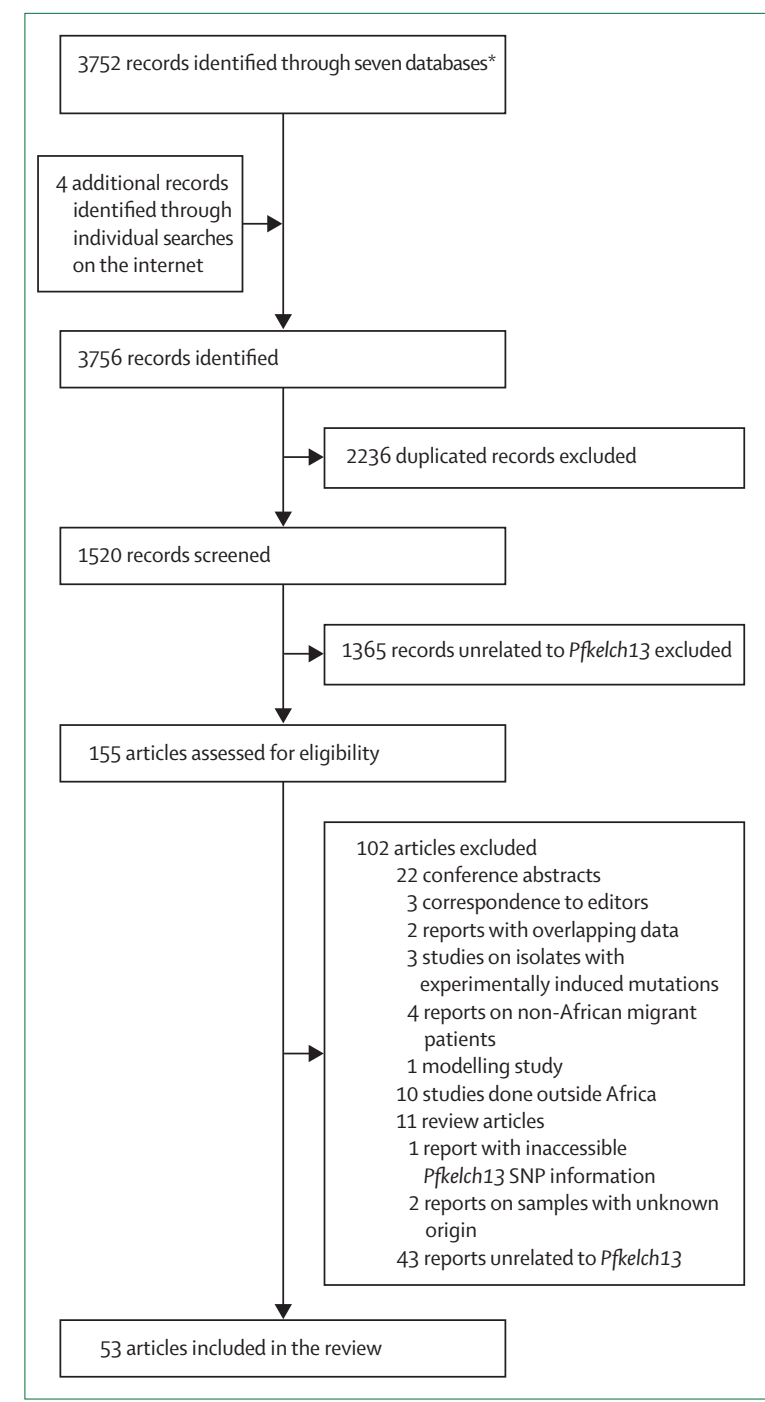

Figure 1: Flowchart of the article selection process

Pfkelch13=Plasmodium falciparum kelch13. SNP=single-nucleotide polymorphisms. *PubMed ( $n=738)$, Scopus ( $n=101)$, Embase ( $n=754)$, African Index Medicus Database ( $n=1207)$, African Journals Online $(n=10)$, Web of Science $(n=926)$, Bioline $(n=16)$.

16 alleles similar to WHO PfART-R markers were also found in 18 isolates $(0.08 \%$ to $5.41 \%$ per sampling site) in seven countries: Gly449Asp and Gly449Ser (similar to Gly449Ala) in Mali, ${ }^{65,7}$ Cys469Trp (similar to Cys469Tyr) in Kenya, ${ }^{42}$ Met476Lys (similar to Met476Ile) in DR Congo, ${ }^{62}$ Ser522Met and Ser522Arg (similar to Ser522Cys) in Togo, Uganda, and Kenya, ${ }^{36,64,78}$ Arg539Ile and Arg539Lys (similar to Arg539Thr) in Senegal and Kenya, ${ }^{36,70}$ Pro553Ile (similar to Pro553Leu) in Senegal,,$^{70}$ Arg561Cys (similar to ArgR561His) in Mali and DR Congo, $97 \pi$ Arg575Gly (similar to Arg575Lys) in Mali, ${ }^{65}$ Asp584Glu, Asp584Asn, and Asp584Tyr (similar to Asp584Val) in Comoros, Mali, and Kenya, ${ }^{36,44,65}$ and Pro667Arg and Pro667Ser (similar to Pro667Thr) in Kenya ${ }^{36}$ (appendix p 11). There has been an increasing trend in reports of PfART-R molecular markers 
Figure 2: Geographical maps displaying proportions of isolates carrying Pfkelch13

with non-synonymous changes in Africa

Pie charts displayed on these maps represent the proportions of isolates

with only synonymous changes in the Pfkelch13 gene, or with at least one non-synonymous change. Overall, isolates with non-synonymous changes were observed in sampling sites located within 30 of 41 surveyed countries in east, west, and central Africa. Moderate-to-high proportions of isolates with non-synonymous changes in the Pfkelch13 sequence were found in east and west Africa. (A) Map showing surveys with only synonymous changes or wild-type isolates.

(B) Map showing surveys with very low $(>0 \%$ to $<5 \%)$ proportions of isolates with non-synonymous Pfkelch13 changes. (C) Low ( $5 \%$ to $<10 \%)$ proportions of isolates with non-synonymous Pfkelch13 changes.

(D) Moderate (10\% to $20 \%$ ) proportions of isolates with non-synonymous Pfkelch13 changes. (E) Low-intermediate (>20\% to $<40 \%$ ) proportions of isolates with non-synonymous Pfkelch13 changes.

(F) Intermediate-high (40 to $<80 \%$ ) proportions of isolates with non-synonymous Pfkelch13 changes.
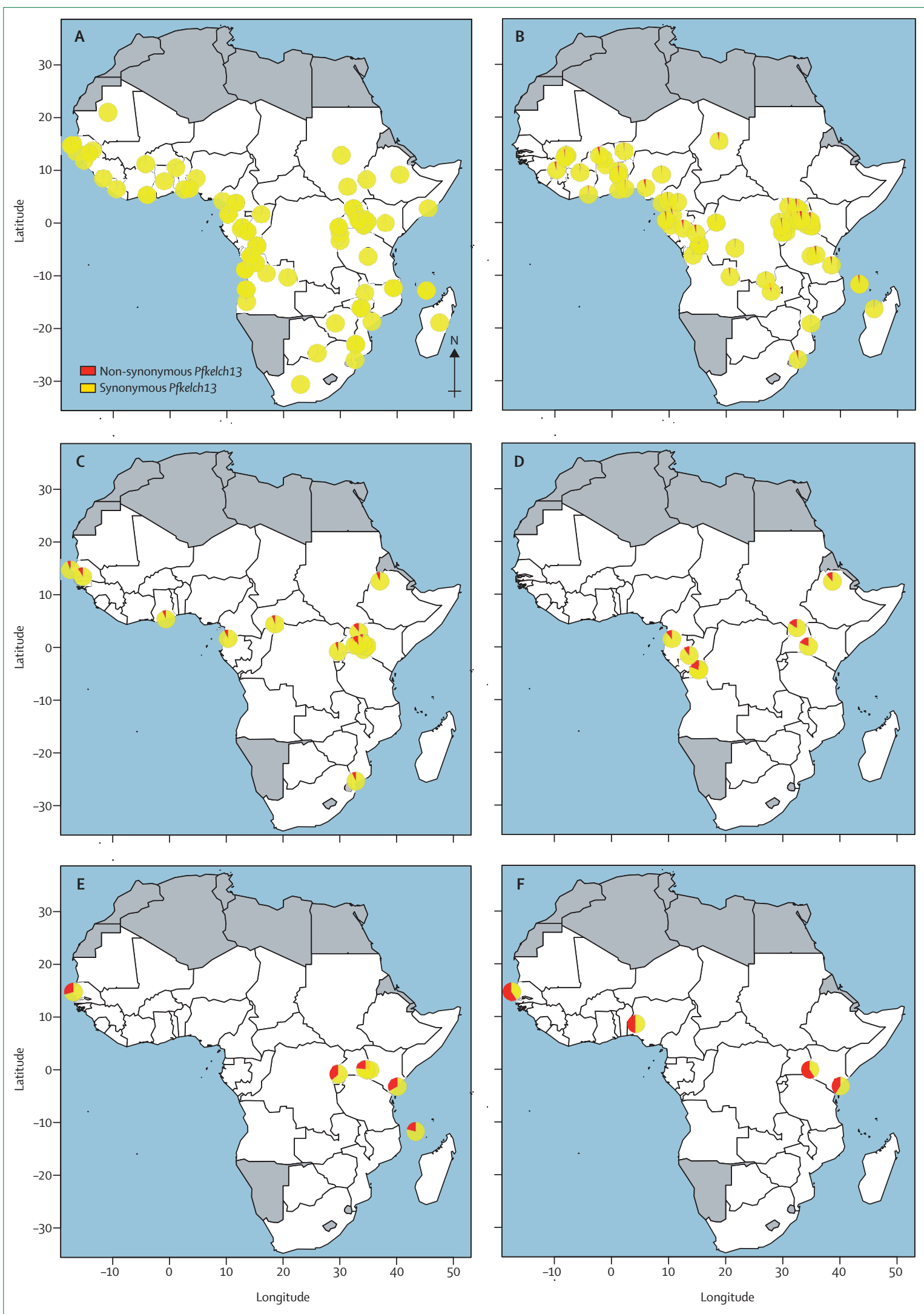
and mutations similar to PfART-R markers in Africa since the first year of reporting in 2014 (figure 5).

\section{Discussion}

This paper is the first comprehensive and systematic review specifically focusing on polymorphisms of the Pfkelch13 gene in Africa. Pfkelch13 has exclusively been explored in sub-Saharan African countries, showing a highly polymorphic structure with most allelic variations located in the propeller domain. Despite low relative frequencies of non-synonymous mutations in this domain across the continent, we identified mutant alleles reported to be associated with diminished artemisinin responsiveness in southeast Asia and several alleles structurally similar to resistance mutations.

The large allelic variation in the Pfkelch13-propeller domain (despite being located in a conserved region of the $P$ falciparum genome) is likely to reflect the adaptation of parasites to selective pressures in Africa (eg, the use of antimalarial drugs). ${ }^{6479}$ Therefore, this information should raise concerns among health policy makers to prevent the use of poor-quality (ie, counterfeit or substandard) artemisinin-based therapies and artemisinin monotherapies in Africa. ${ }^{79,80}$ Sub-therapeutic antimalarial drug concentrations would provide ideal conditions for selecting Pfkelch13-propeller mutants, among which PfART-R mutants could emerge. ${ }^{18,81}$ This hypothesis is supported by the generation of Pfkelch13-propeller mutations in isolates with an African genetic background that underwent in-vitro experimental artemisinin selective pressure, and the subsequent appearance of the PfART-R phenotype. ${ }^{14,82}$

Unlike the high frequency of non-synonymous mutations in southeast Asia (with proportions ranging from intermediate to fixation levels among sequenced parasites), Pfkelch13 non-synonymous mutations in Africa occur at very low relative frequencies, despite presenting high allelic variation. The delayed introduction

Figure 3: Molecular map of alleles in the Pfkelch13-propeller domain of African isolates This figure shows allelic variations of amino acid residues within blades (1-6) of the Pfkelch13-propeller domain (after position 440) based on the Plasmodium falciparum 3D7 reference sequence. Of the 280 loci in this sequence, 150 were polymorphic with synonymous and non-synonymous alleles. Loci reported with either a wild-type residue or synonymous mutations are shaded in yellow. Colours in cells identify whether the non-synonymous mutation is classified as PfART-R marker (red), a mutation mimicking a known PfART-R (dark blue), and a non-PfART-R marker (light blue). Single-letter abbreviations for the amino acid residues are as follows: $A=$ alanine, $C=$ cysteine $\mathrm{D}=$ aspartic acid, $\mathrm{E}=$ glutamic acid, $\mathrm{F}=$ phenylalanine, $\mathrm{G}=$ glycine, $\mathrm{H}=$ histidine, I=isoleucine, $\mathrm{K}=$ lysine, $\mathrm{L}=$ leucine, $\mathrm{M}=$ methionine, $\mathrm{N}=$ aspargine, $\mathrm{P}=$ proline $\mathrm{Q}=$ glutamine, $\mathrm{R}=$ arginine, $\mathrm{S}=$ serine, $\mathrm{T}=$ threonine, $\mathrm{V}=$ valine, $\mathrm{W}=$ tryptophan, and $\mathrm{Y}=$ tyrosine. In mutants, reference amino acids at specified locations were

substituted with other amino acids-eg, M476I indicates that methionine at codon position 476 was replaced by isoleucine. The asterisk $\left({ }^{*}\right)$ shows a substitution of a reference amino acid residue by a stop-codon within the mutant (nonsense mutation). The underscore (_) shows deletion of a reference amino acid residue at a given codon-position within the mutant (deletion). PfART-R=Plasmodium falciparum artemisinin resistance. \begin{tabular}{|l|l|l|l|l|l|l|l|l|l|l|}
\hline 1 & P441 & F442L & M442V & P443 & L444 & V445 & F4461 & C447 & 1448L & 1448M \\
\hline
\end{tabular}

\begin{tabular}{|c|c|c|c|c|c|c|c|c|c|}
\hline G449D & G449S & G450 & F451 & D452 & G453 & V454A & V454I & E455 & Y456 \\
\hline L457I & N458 & S459S & M460 & E461 & L462 & L463S & $\mathrm{D} 464 \mathrm{H}$ & D464N & D464Y \\
\hline D464D & $1465 T$ & S466l & Q467H & Q467Q & Q468R & Q468Q & C469w & C469Y & C469C \\
\hline W470* & R471R & M4721 & M472V & C473F & C473_ & C473Y & T474T & & \\
\hline
\end{tabular}

2

\begin{tabular}{|l|l|l|l|l|l|l|l|l|l|}
\hline P475 & M4761 & M476K & S477Y & S477S & T478P & T478T & K479 & K480R & A481 \\
\hline Y482S & F483 & G484R & S485 & A486 & V487I & L488S & N489S & S489K & N489N \\
\hline N490H & N490S & F491S & L492 & Y493Y & V494A & V494I & V494L & F495L & G496G \\
\hline G496S & G497 & N498I & N499D & N4990 & Y500Y & D501G & Y502 & K503K & A504G \\
\hline A504T & A504V & L505F & L505S & F506 & E507E & T508 & E509D & E509E & E509G \\
\hline V510M & V510_ & V510V & Y511 & D512N & R513R & R513S & L514 & R515K & D516 \\
\hline \hline V517 & W518C & Y519C & V520A & S521 & S522C & S522M & S522R & S522S & N523T \\
\hline L524 & N525 & I526M & \multicolumn{10}{|c|}{} \\
\hline
\end{tabular}

3

\begin{tabular}{|c|c|c|c|c|c|c|c|c|c|}
\hline P527 & R528 & R529K & N530l & N530K & N530Y & N5311 & $\mathrm{N} 531 \mathrm{~N}$ & N531Y & C532S \\
\hline G533C & G533S & G533V & V534A & V534I & V534L & T535M & T535T & S536L & N537 \\
\hline G538G & R539I & R539K & R539R & 1540 & Y541 & $\mathrm{C} 542 \mathrm{G}$ & C542Y & 1543 & G544R \\
\hline G545E & Y546 & D547 & G548D & G548G & G548S & S549P & $\mathrm{S} 550$ & 1551 & I552M \\
\hline $\mathrm{S} 552 \mathrm{C}$ & P5531 & P553L & P553P & A554S & $\mathrm{N} 554 \mathrm{H}$ & N554K & N5540 & N554S & V555A \\
\hline V555L & E556K & E556V & A557S & Y558C & $\mathrm{Y} 558 \mathrm{H}$ & D559N & $\mathrm{H} 560 \mathrm{~L}$ & R561C & M562T \\
\hline K563 & A564 & W565* & V5661 & E567E & E567K & V568G & A569G & A569S & A569T \\
\hline P570L & L571 & N572 & T573A & & & & & & \\
\hline
\end{tabular}

\begin{tabular}{|l|l|l|l|l|l|l|l|l|l|}
\hline P574L & R575G & R575R & S576L & S577P & A578D & A578S & M579 & C580 & V581F \\
\hline V581V & A582 & F583L & F583S & F583Y & D584E & D584N & D584Y & N585K & K586 \\
\hline N587K & Y588C & L5891 & V5891 & V589V & I590F & G591D & G592E & G592G & G592R \\
\hline G592V & T593 & N594 & G595S & E596G & E596K & E596R & R5971 & R597R & L598F \\
\hline \hline L598L & N599 & 1601T & E602D & E602E & E602 & V603 & Y604 & E605G & E606G \\
\hline K607 & M608L & B609B & N609L & N609N & K610K & W611_ & E612D & E612G & Q613E \\
\hline Q613H & \multicolumn{10}{|c|}{} \\
\hline
\end{tabular}

\begin{tabular}{|l|l|l|l|l|l|l|l|l|l|l|}
\hline 5 & F614L & P615S & Y616N & A617T & A617V & L618 & L619S & E620K & A621A & A621S \\
\hline
\end{tabular}

\begin{tabular}{|l|l|l|l|l|l|l|l|l|l|l|}
\hline R622G & R6221 & S623 & S624 & G625R & A626P & A626S & A626T & A626V & A627 \\
\hline F628L & N629T & Y630P & L631 & N632 & Q633R & Q633* $^{*}$ & I634 & Y635C & V636 \\
\hline V637A & V637D & V6371 & V637_ & G638R & G639C & G639S & G639V & I640V & D641G \\
\hline \hline N642 & E643 & H644 & N645 & I646 & L647 & D648H & S649 & V650 & E651 \\
\hline Q652 & Y653 & Q654Q & P655 & F6561 & N657 & K658 & R659 & W660C & Q661 \\
\hline F662 & L663 & N664 & G665C & G665S & & & & & \\
\hline
\end{tabular}

6

\begin{tabular}{|l|l|l|l|l|l|l|l|l|l|}
\hline V666A & V6661 & V666V & T667R & T6675 & E668 & K669K & K670 & M671 & N672 \\
\hline F673 & G674 & A675V & A676S & A676V & T677A & T677K & L678V & S679 & D680N \\
\hline S681 & Y682 & I683 & 1684 & T685 & G686 & G687 & E688 & N689 & G690C \\
\hline G690G & E691 & V692 & L693 & N694 & S695 & C696 & H697 & F698F & F699 \\
\hline \hline S700 & P701 & D702 & T703 & N704 & E705 & W706 & Q707 & L708 & G709 \\
\hline P710 & S711 & L712 & L713 & V714 & P715 & R716 & F717 & G718 & H719 \\
\hline S720 & V721 & L722 & I723 & A724 & N725 & I726 & \multicolumn{1}{|l}{} \\
\hline
\end{tabular}

$\square$ Wild-type residue or synonymous mutation

$\square$ Non-synonymous mutation not classified by WHO as a PfART-R marke

$\square$ Non-synonymous mutation classified by WHO as a PfART-R marke

$\square$ Non-synonymous mutation mimicking a PfART-R marker 

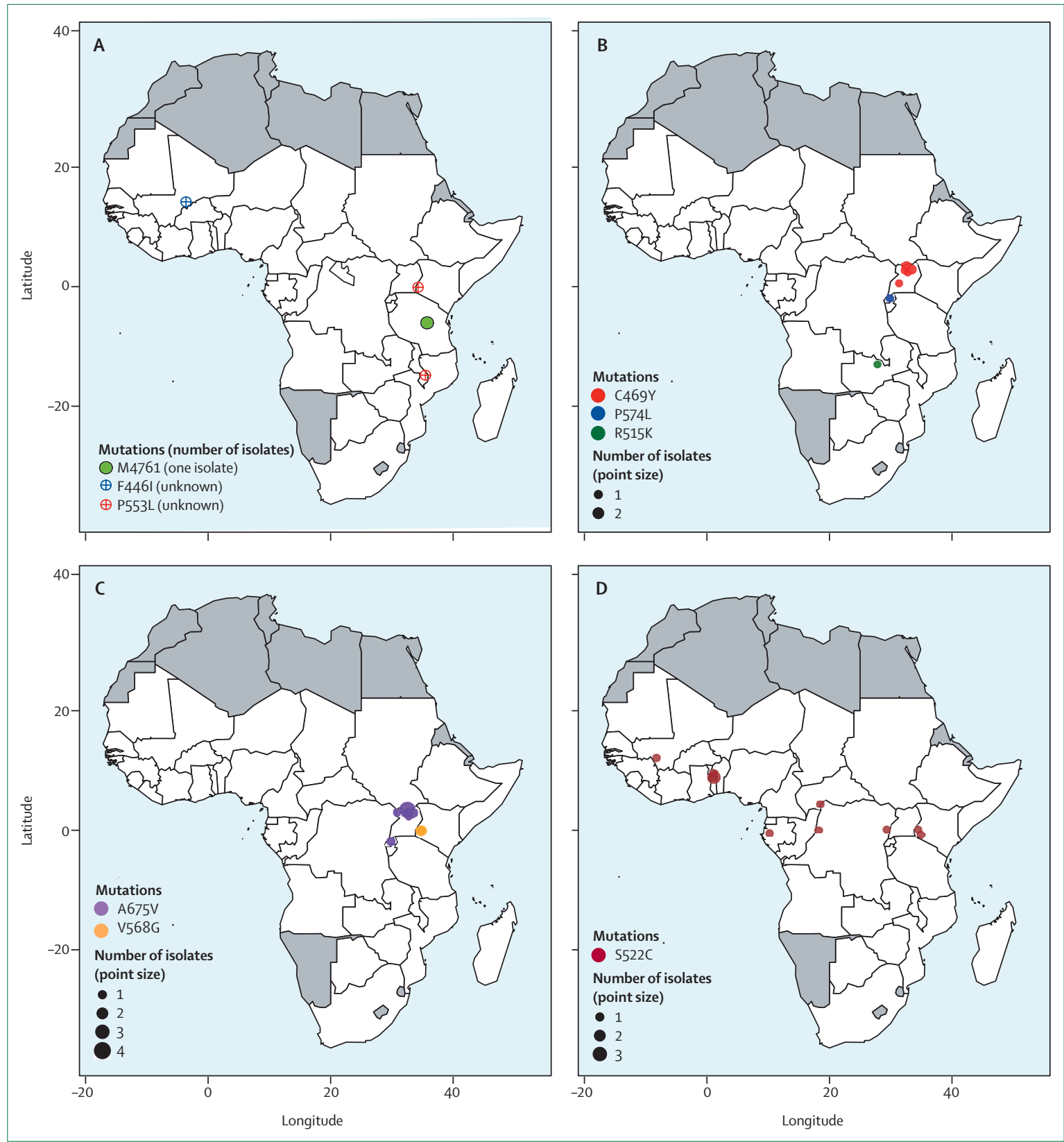

Figure 4: Maps displaying sampling sites that recorded isolates carrying WHO PfART-R markers in Africa

Points plotted on these maps represent isolates carrying mutations associated with PfART-R in southeast Asia according to the WHO 2018 list of PfART-R markers. ${ }^{17}$ The point size is proportional to the number of isolates that were identified at each sampling location whenever it is known. Mutations with an unknown number of isolates are shown as crossed-out circles. (A) Map showing surveys that detected PfART-R markers validated by WHO in at least one isolate in four countries, including F4461 in Mali, M476I in Tanzania, and P553L in Kenya and Malawi. (B-D) Maps showing surveys that recorded WHO candidate PfART-R markers including C469Y in Uganda, R515K in Zambia, S522C in Togo, Central African Republic, Gabon, DRC, Uganda, and Kenya, V568G in Kenya, P574L in Rwanda, and $\mathrm{A} 675 \mathrm{~V}$ in Uganda and Rwanda. Single-letter abbreviations for the amino acid residues are as follows: $\mathrm{A}=$ alanine, $\mathrm{C}=\mathrm{cysteine}, \mathrm{F}=$ phenylalanine, $\mathrm{G}=$ glycine, $\mathrm{I}=$ isoleucine, $\mathrm{K}=$ lysine, $\mathrm{L}=$ leucine, $\mathrm{M}=$ methionine, $\mathrm{P}=$ proline, $\mathrm{S}=$ serine, $\mathrm{V}=\mathrm{valine}$; and $\mathrm{Y}=$ tyrosine. Mutations show that reference amino acids at specified locations were substituted with other amino acids; for example, M4761 indicates that a methionine at codon-position 476 was replaced by an isoleucine. PfART-R=Plasmodium falciparum artemisinin resistance.

of artemisinin in Africa between 2000 and 2005, associated with a shorter time of drug pressure by comparison with the early use of artemisinin in southeast Asia since the 1970s, could explain why there is a scarcity of isolates with non-synonymous mutations in Africa ${ }^{83}$ Moreover, multi-country studies done in Africa, southeast Asia, and South America had suggested that non-synonymous Pfkelch13 mutations reported in African countries could be due to local adaptation rather than to importation from Asia, because alleles were mostly Africa-specific 


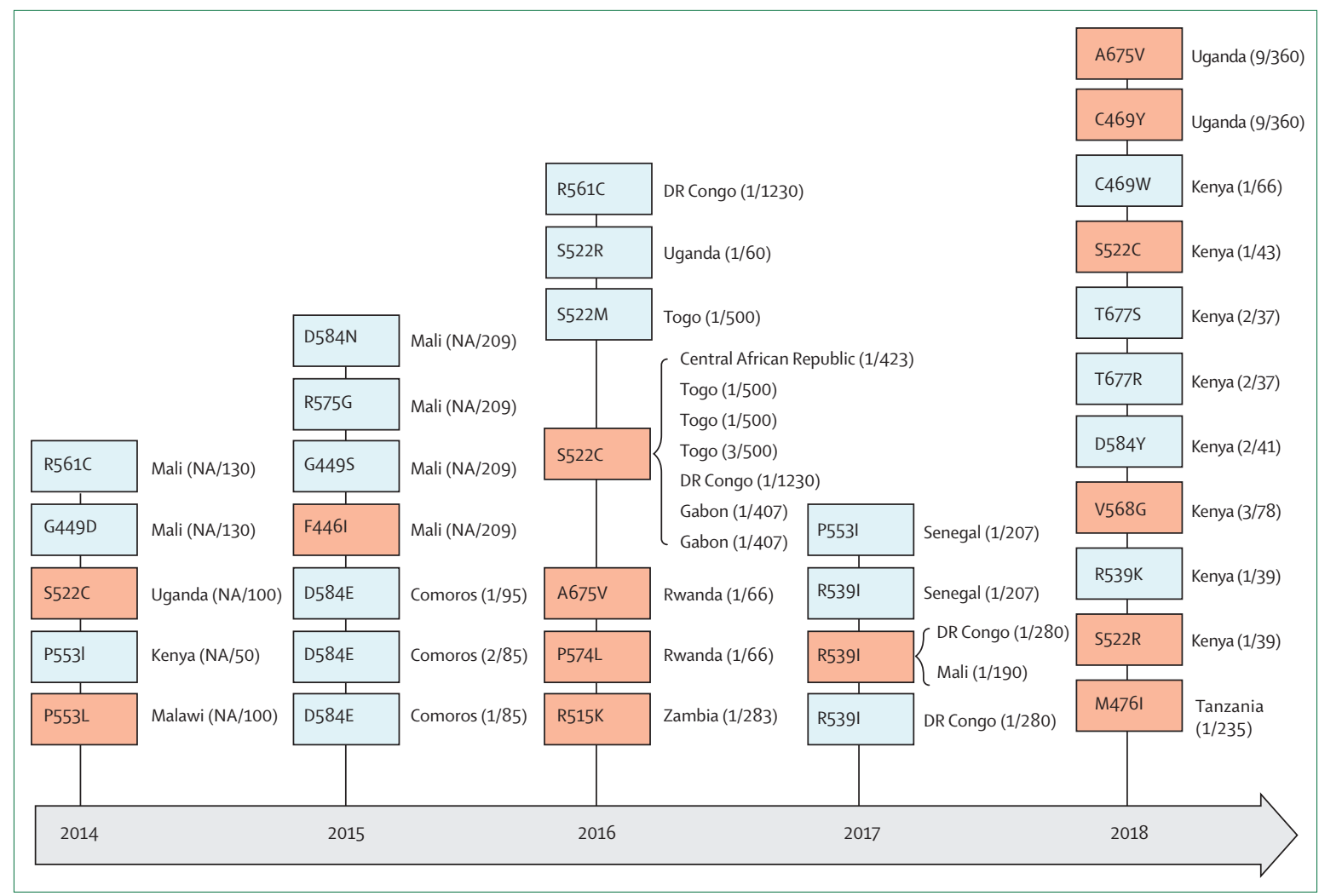

Figure 5: Evolution in the reporting of PfART-R molecular markers during 2014-18

Graph shows the discovery of different PfART-R markers (shaded in red) and mutations similar to PfART-R markers (shaded in dark blue), over the time in Africa. The years 2014-18 indicate the publication year of the articles reporting mutants. Single-letter abbreviations for the amino acid residues are as follows: $A=a l a n i n e$, $\mathrm{C}=$ cysteine, $\mathrm{D}=$ =aspartic acid, $\mathrm{E}=$ glutamic acid, $\mathrm{F}=$ phenylalanine, $\mathrm{G}=$ glycine, $\mathrm{I}=$ isoleucine, $\mathrm{K}=$ lysine, $\mathrm{L}=$ leucine, $\mathrm{M}=$ methionine, $\mathrm{N}=$ aspargine, $\mathrm{P}=$ proline, $\mathrm{R}=$ arginine, $\mathrm{S}=$ serine, $\mathrm{T}=$ threonine, $\mathrm{V}=$ valine, $\mathrm{W}=$ tryptophan, and $\mathrm{Y}=$ tyrosine. In mutant alleles, reference amino acids at specified locations were substituted with other amino acids; for example, M476I shows that methionine at codon-position 476 was replaced by isoleucine. PfART-R=Plasmodium falciparum artemisinin resistance.

and structurally different from those observed in southeast Asia ..$^{9,18,77}$

The WHO list of African Pfkelch13 alleles included nine non-synonymous mutations of the total 31 molecular markers of PfART-R based on studies done mainly in southeast Asia. ${ }^{17}$ Six of these mutations, namely Phe446Ile, Met476Ile, Pro553Leu, Val568Gly, Pro574Leu, and Ala675Val, were reported to be strongly associated with delayed parasite clearance in vivo. ${ }^{14,18,84,85}$ However, only Phe446Ile, Met476Ile, and Pro533Leu have shown a decreased response to artemisinin in vitro; they are being considered as validated artemisinin resistance markers by WHO. ${ }^{14,16,17,86}$ Ala675Val, although not listed as a validated marker, has shown an altered response to artemisinin in vitro in one clinical isolate from Uganda. ${ }^{45}$ Phe446Ile was only found in west Africa (Mali), whereas Ala675Val, Met476Ile, Pro553Leu, Pro574Leu, and Val568Gly were reported in the Great Lakes region in east Africa (Kenya, Uganda, Malawi, Zambia, Tanzania, and Rwanda). The other three mutations identified in African isolates (Ser522Cys, Cys469Tyr, and Arg515Lys) could also be related to causing slow parasite clearance by artemisinin; however, according to the WHO, there is no significant evidence for this association because of the low number of these mutants in surveys. ${ }^{17}$ The Ser522Cys mutation that was found in several African countries (Mali, Gabon, Central African Republic, Togo, DR Congo, and Kenya), ${ }^{9,42,69,78}$ had been correlated with delayed parasite clearance in one (from southeast Asia) of the three unique mutant isolates that have been assessed in vivo (one isolate being from DR Congo). ${ }^{18,87}$ Similarly, two Cys469Tyr mutants have been linked to causing low parasite clearance and day 3 positive parasitaemia after treatment with artemisinin in southeast Asia. ${ }^{87,88}$ However, in our systematic review, the unique African isolate with this mutation (from Uganda) assessed in vitro did not show a reduced response to artemisinin. ${ }^{45}$ Unlike Ser522Cys and Cys469Tyr, the data accumulated for the Arg515Lys mutation deserves to be taken into account in future lists of PfART-R markers as more than ten mutants from southeast Asia have been consistently associated with delayed parasite clearance. . $5,87,89$

Nine non-synonymous mutations that are classified by the WHOas eithervalidated, candidate, or suspectedartemisinin resistance markers, and 245 other Pfkelch13-propeller mutations were registered during the writing of this 
systematic review. Most of them have never been experimentally explored because they did not fulfil the criteria for further functional exploration (eg, high frequency of new alleles with non-synonymous mutation, evidence of allele dissemination, or preliminary association with clinical resistance). ${ }^{9} 16$ amino acid substitutions among the unexplored mutations found in this review are structurally close to the WHO PfART-R mutations-ie, Gly449Asp, Gly449Ser, Cys469Trp, Met476Lys, Ser522Met, Ser522Arg, Arg539Ile, Arg539Lys, Prp553Ile, Arg561Cys, Arg575Gly, Asp584Glu, Asp584Asn, Asp584Tyr, Thr677Arg, and Thr677Ser. For instance, Gly449Asp and Gly449Ser observed in Mali $^{65}$ are able to resemble the PfART-R mutation Gly449Ala. They occur on a locus known to be accessible to glycine (Gly449) and establish a network of hydrogen bonds for the Pfkelch13 protein function. ${ }^{90}$ These two African alleles could be structurally unfavourable to the structure of the Pfkelch13 propeller domain, resulting in potential markers for PfART-R..$^{90}$ Molecular modelling can thus provide more information about the phenotypic effect of unexplored mutations on the Pfkelch13 protein structure; ${ }^{9,90}$ however, conventional research (in vitro or in vivo studies using either transgenic or clinical parasites) is always required to confirm their functional importance. Until the functional effect of these mutations that are similar to PfART-R markers is clarified, we suggest that they are included in any molecular surveillance of PfART-R in Africa when possible.

It is important to mention that African parasites might have their own genetic background preference to select PfART-R that would differ from parasites in southeast Asia. ${ }^{8,91}$ The discovery of Pfcoronin in 2018 as a second PfART-R gene suggests that non-Pfkelch13 types of resistance could independently emerge in natural settings in Africa. ${ }^{92-94}$ Pfcoronin mutants associated with PfART-R were selected among parasites of Senegalese origin, using the same in-vitro selection experiment that had related Pfkelch13 mutations to reduced artemisinin susceptibility in Tanzanian parasites. ${ }^{14,90,92}$ Although the Pfcoronin mutations are not yet detected in clinical isolates, their structural similarity with Pfkelch13 provides insights into the molecular mechanisms of artemisinin resistance. ${ }^{93-95}$ Tracking the emergence of Pfkelch13 mutant parasites in Africa is very important, but further research is needed to identify other possible PfART-R genes. ${ }^{91}$

Our systematic review has several limitations. First, no information is available from non-endemic malaria areas-eg, the Maghreb countries (eg, Algeria, Libya, Morocco, Egypt, Western Sahara, and Tunisia)-that have a lot of migration from sub-Saharan Africa, where the PfART-R threat might also exist. Future surveys should fill in the gaps of the existing map. Second, the heterogeneity in survey designs, sampling methods (eg, convenience samples), and analytical approaches in individual studies (eg, different lengths of analysed Pfkelch13 sequences, different sampling time in surveys) did not allow pooling of reports for a standard meta-analysis. Third, primary information (eg, the precise collection time) was very variable or not available in some surveys, hindering the possibility of analysing temporal trends. Instead, we assessed the temporal trend of reporting PfART-R mutations. Fourth, the inaccuracy in the number of isolates carrying alleles in some reviewed studies and mutations reported with unknown origin that were excluded from the review could have led to an underestimation of allele frequencies (eg, Pro553Leu in Malawi ${ }^{7}$ or Phe446Ile in Mali $\left.{ }^{65}\right)$. For these studies, the frequency of sites with these mutations was reported without assigning allele frequencies. Finally, our review did not include studies reporting migrating patients who returned to their home countries from Africa with isolates carrying Pfkelch13 mutations. For instance, three PfART-R markers (Cys580Tyr, Met579Ile, and Arg539Thr) found in isolates of patients returning to Asia from Africa were excluded from this Review. ${ }^{96,97}$ These mutations that favour PfART-R were probably acquired outside Africa as no autochthonous patients had these mutations, despite published reports extensively discussing that acquisition in Africa cannot be ruled out.

Despite the limitations above, we believe that our systematic review provides a valuable baseline reference for building and strengthening surveillance activities in African countries to prevent the emergence and later spread of PfART-R. The message in this paper is not a lack of confidence in ACTs, which are still very effective in Africa, but it is more a call for vigilance and increased surveillance efforts against possible PfART-R emergence locally. Therefore, proactive surveillance using PfART-R molecular markers and timely consolidation and interpretation of surveillance findings can contribute to decision making to further sustain the effectiveness of artemisinin in Africa. Any possible introduction by known PfART-R alleles, temporal increase in the frequency of autochthonous alleles, and expansion of nonsynonymous mutations similar to PfART-R markers should be targeted by surveillance efforts. Enhanced research efforts (ie, more funding and field exploration) are required to uncover the functional importance of other Pfkelch13-propeller mutations and to identify other possible PfART-R genes like Pfcoronin.

\section{Contributors}

NS and NKK conceived the study. NKK, ET-K, AR-A, and BD wrote the protocol. NKK, DYM, AR-A, ET-K, VPT, and YY did the literature search, acquired the aggregate data, screened records, and extracted the data. NKK, ET-K and AR-A did the data analyses and made figures. NKK, ET-K, AR-A, VPT, BD, and NS wrote the first draft of the manuscript. DMM, GLM, DYM, PDM, M-PH, YY, and EOW revised the first draft of the manuscript. NS had full access to all the data in the study and had final responsibility for the decision to submit for publication. All authors provided conceptual input, and revised and approved of the final version of the manuscript.

Declaration of interests

We declare no competing interests.

\section{Acknowledgments}

This systematic review was funded by the Belgian Cooperation Agency through the Académie de Recherche et d'Enseignement Supérieur. 
The funder of the study had no role in study design, data collection, data analysis, data interpretation, or writing of the report.

\section{References}

1 WHO. World malaria report 2018. Nov 19, 2018. https://www.who. int/malaria/publications/world-malaria-report-2018/en/ (accessed March 16, 2019).

2 Bhatt S, Weiss D, Cameron E, et al. The effect of malaria control on Plasmodium falciparum in Africa between 2000 and 2015. Nature 2015; 526: 207-11.

3 Menard D, Dondorp A. Antimalarial drug resistance: a threat to malaria elimination. Cold Spring Harb Perspect Med 2017; 7: a025619.

4 Noedl H, Se Y, Schaecher K, et al. Evidence of artemisinin-resistant malaria in western Cambodia. N Engl J Med 2008; 359: 2619-20.

5 Dondorp AM, Nosten F, Yi P, et al. Artemisinin resistance in Plasmodium falciparum malaria. N Engl J Med 2009; 361: 455-67.

6 Amaratunga C, Sreng S, Suon S, et al. Artemisinin-resistant Plasmodium falciparum in Pursat province, western Cambodia: a parasite clearance rate study. Lancet Infect Dis 2012; 12: 851-58.

7 Loy DE, Liu W, Li Y, et al. Out of Africa: origins and evolution of the human malaria parasites Plasmodium falciparum and Plasmodium vivax. Int J Parasitol 2017; 47: 87-97.

8 Mita T, Tachibana S-I, Hashimoto M, Hirai M. Plasmodium falciparum kelch 13: a potential molecular marker for tackling artemisinin-resistant malaria parasites. Expert Rev Anti Infect Ther 2016; 14: 125-35.

9 Ménard D, Khim N, Beghain J, et al. A worldwide map of Plasmodium falciparum K13-propeller polymorphisms. N Engl J Med 2016; 374: 2453-64.

10 Wells TN, Van Huijsduijnen RH, Van Voorhis WC. Malaria medicines: a glass half full? Nat Rev Drug Discov 2015 14: $424-42$.

11 WHO. Methods for surveillance of antimalarial drug efficacy. November, 2009. https:/www.who.int/malaria/publications/ atoz/9789241597531/en/ (accessed March 16, 2019).

12 Plowe C. Antimalarial drug resistance in Africa: strategies for monitoring and deterrence. Curr Top Microbiol Immunol 2005; 295: 55-79.

13 Flegg JA, Guerin PJ, White NJ, Stepniewska K. Standardising the measurement of parasite clearance in falciparum malaria: the parasite clearance estimator. Malar J 2011; 10: 339.

14 Ariey F, Witkowski B, Amaratunga C, et al. A molecular marker of artemisinin-resistant Plasmodium falciparum malaria. Nature 2014; 505: 50-55.

15 Saralamba S, Pan-Ngum W, Maude RJ, et al. Intrahost modeling of artemisinin resistance in Plasmodium falciparum. Proc Natl Acad Sci USA 2011; 108: 397-402.

16 Straimer J, Gnädig NF, Witkowski B, et al. K13-propeller mutations confer artemisinin resistance in Plasmodium falciparum clinical isolates. Science 2015; 347: 428-31.

17 WHO. Artemisinin resistance and artemisinin-based combination therapy efficacy: status report. 2018. https://apps.who.int/iris/ handle/10665/274362 (accessed March 20, 2019).

18 Ashley EA, Dhorda M, Fairhurst RM, et al. Spread of artemisinin resistance in Plasmodium falciparum malaria. N Engl J Med 2014; 371: 411-23.

19 Moher D, Liberati A, Tetzlaff J, Altman D. Preferred reporting items for systematic reviews and meta-analyses: the PRISMA statement. PLoS Med 2009; 6: e1000097.

20 Bernardo WM. PRISMA statement and PROSPERO. Int Braz J Urol 2017; 43: 383-84.

21 Stang A. Critical evaluation of the Newcastle-Ottawa scale for the assessment of the quality of nonrandomized studies in metaanalyses. Eur Journal Epidemiol 2010; 25: 603-05.

22 den Dunnen JT, Antonarakis SE. Nomenclature for the description of human sequence variations. Hum Genet 2001; 109: 121-24.

23 Ogino S, Gulley ML, den Dunnen JT, Wilson RB. Standard mutation nomenclature in molecular diagnostics: practical and educational challenges. J Mol Diagn 2007; 9: 1-6.

24 Wickham H. ggplot2: elegant graphics for data analysis. New York: Springer-Verlag, 2016.

25 Kahle D, Wickham H. ggmap: spatial Visualisation with ggplot2. R J 2013; 5: 144-161.
26 Keitt TH, Bivand R, Pebesma E, Rowlingson B. rgdal: bindings for the Geospatial Data Abstraction Library. https://cran.r-project.org/ web/packages/rgdal/index.html (accessed Jan 5, 2019).

27 R Core Team. R: a language and environment for statistical computing. 2018. https://scholar.google.com/scholar?hl=fr\&as_sdt= $0 \% 2 \mathrm{C} 5 \& \mathrm{q}=\mathrm{R}+$ Core+Team. $+\mathrm{R} \% 3 \mathrm{~A}+\mathrm{a}+$ language+and+environment+ for+statistical+computing.+2018.+http\%3A\%2F\%2Fwww.R-project. org\%2F\&btnG $=$ (accessed Sept 11, 2020).

28 Apinjoh TO, Mugri RN, Miotto O, et al. Molecular markers for artemisinin and partner drug resistance in natural Plasmodium falciparum populations following increased insecticide treated net coverage along the slope of mount Cameroon: cross-sectional study. Infect Dis Poverty 2017; 6: 136.

29 Asua V, Vinden J, Conrad MD, et al. Changing molecular markers of antimalarial drug sensitivity across Uganda. Antimicrob Agents Chemother 2019; 63: e01818-18.

30 Balikagala B, Mita T, Ikeda M, et al. Absence of in vivo selection for K13 mutations after artemether-lumefantrine treatment in Uganda. Malar J 2017; 16: 23.

31 Bayih AG, Getnet G, Alemu A, Getie S, Mohon AN, Pillai DR. A unique Plasmodium falciparum K13 gene mutation in northwest Ethiopia. Am J Trop Med Hyg 2016; 94: 132-35.

32 Boussaroque A, Fall B, Madamet M, et al. Emergence of mutations in the K13 propeller gene of Plasmodium falciparum isolates from Dakar, Senegal, in 2013-2014. Antimicrob Agents Chemother 2016; 60: 624-27.

33 Conrad MD, Bigira V, Kapisi J, et al. Polymorphisms in K13 and falcipain-2 associated with artemisinin resistance are not prevalent in Plasmodium falciparum isolated from Ugandan children. PloS One 2014; 9: e105690.

34 Cooper RA, Conrad MD, Watson QD, et al. Lack of artemisinin resistance in Plasmodium falciparum in Uganda based on parasitological and molecular assays. Antimicrob Agents Chemother 2015; 59: 5061-64.

35 Dama S, Niangaly H, Ouattara A, et al. Reduced ex vivo susceptibility of Plasmodium falciparum after oral artemetherlumefantrine treatment in Mali. Malar J 2017; 16: 59.

36 de Laurent ZR, Chebon LJ, Ingasia LA, et al. Polymorphisms in the K13 gene in Plasmodium falciparum from different malaria transmission areas of Kenya. Am J Trop Med Hyg 2018; 98: 1360-66.

37 Dieye B, Affara M, Sangare L, et al. West Africa international centers of excellence for malaria research: drug resistance patterns to artemether-lumefantrine in Senegal, Mali, and The Gambia. American J Trop Med Hyg 2016; 95: 1054-60.

38 Djaman JA, Olefongo D, Ako AB, et al. Molecular epidemiology of malaria in Cameroon and Côte d'Ivoire. XXXI. Kelch 13 propeller sequences in Plasmodium falciparum isolates before and after implementation of artemisinin-based combination therapy. Am J Trop Med Hyg 2017; 97: 222-24.

39 Escobar C, Pateira S, Lobo E, et al. Polymorphisms in Plasmodium falciparum K13-propeller in Angola and Mozambique after the introduction of the ACTs. PLoS One 2015 10: e0119215.

40 Guerra M, Neres R, Salgueiro P, et al. Plasmodium falciparum genetic diversity in continental Equatorial Guinea before and after introduction of artemisinin-based combination therapy. Antimicrob Agents Chemother 2017; 61: e02556-15.

41 Gupta H, Macete E, Bulo H, et al. Drug-resistant polymorphisms and copy numbers in Plasmodium falciparum, Mozambique, 2015. Emerg Infect Dis 2018; 24: 40-48.

42 Hemming-Schroeder E, Umukoro E, Lo E, et al. Impacts of antimalarial drugs on Plasmodium falciparum drug resistance markers, Western Kenya, 2003-2015. Am J Trop Med Hyg 2018; 98: 692-99.

43 Ogouyèmi-Hounto $A$, Damien $G$, Deme $A B$, et al. Lack of artemisinin resistance in Plasmodium falciparum in northwest Benin after 10 years of use of artemisinin-based combination therapy. Parasite 2016; 23: 28.

44 Huang B, Deng C, Yang T, et al. Polymorphisms of the artemisinin resistant marker (K13) in Plasmodium falciparum parasite populations of Grande Comore Island 10 years after artemisinin combination therapy. Parasit Vectors 2015; 8: 634

45 Ikeda M, Kaneko M, Tachibana S-I, et al. Artemisinin-resistant 
Plasmodium falciparum with high survival rates, Uganda, 2014-2016. Emerg Infect Dis 2018; 24: 718-26.

46 Isozumi R, Uemura H, Kimata I, et al. Novel mutations in K13 propeller gene of artemisinin-resistant Plasmodium falciparum. Emerg Infect Dis 2015; 21: 490-92.

47 Kakolwa MA, Mahende MK, Ishengoma DS, et al. Efficacy and safety of artemisinin-based combination therapy, and molecular markers for artemisinin and piperaquine resistance in mainland Tanzania. Malar J 2018; 17: 369.

48 Kamau E, Campino S, Amenga-Etego L, et al. K13-propeller polymorphisms in Plasmodium falciparum parasites from sub-Saharan Africa. J Infect Diseases 2015; 211: 1352-55.

49 Kiaco K, Teixeira J, Machado M, do Rosário V, Lopes D. Evaluation of artemether-lumefantrine efficacy in the treatment of uncomplicated malaria and its association with pfmdr1, pfatpase6 and K13-propeller polymorphisms in Luanda, Angola. Malar J 2015; 14: 504.

50 Koukouikila-Koussounda F, Jeyaraj S, Nguetse CN, et al. Molecular surveillance of Plasmodium falciparum drug resistance in the Republic of Congo: four and nine years after the introduction of artemisinin-based combination therapy. Malar J 2017; 16: 155

51 Leroy D, Macintyre F, Adamy M, et al. High proportion of multiple copies of Plasmodium falciparum plasmepsin-2 gene in African isolates: is piperaquine resistance emerging in Africa? bioRxiv 2018; published online July 3. https://www.biorxiv.org/ content/10.1101/361204v1 (preprint).

52 Li J, Chen J, Xie D, et al. Limited artemisinin resistance-associated polymorphisms in Plasmodium falciparum K13-propeller and PfATPase6 gene isolated from Bioko Island, Equatorial Guinea. Int J Parasitol Drugs Drug Resist 2016; 6: 54-59.

53 Ljolje D, Dimbu PR, Kelley J, et al. Prevalence of molecular markers of artemisinin and lumefantrine resistance among patients with uncomplicated Plasmodium falciparum malaria in three provinces in Angola, 2015. Malar J 2018; 17: 84.

54 Lucchi NW, Komino F, Okoth SA, et al. In vitro and molecular surveillance for antimalarial drug resistance in

Plasmodium falciparum parasites in western Kenya reveals sustained artemisinin sensitivity and increased chloroquine sensitivity. Antimicrob Agents Chemother 2015; 59: 7540-47.

55 Plucinski MM, Dimbu PR, Macaia AP, et al. Efficacy of artemether-lumefantrine, artesunate-amodiaquine, and dihydroartemisinin-piperaquine for treatment of uncomplicated Plasmodium falciparum malaria in Angola, 2015. Malar J 2017; 16: 62 .

56 Mayengue PI, Niama RF, Kouhounina Batsimba D, et al. No polymorphisms in K13-propeller gene associated with artemisinin resistance in Plasmodium falciparum isolated from Brazzaville, Republic of Congo. BMC Infect Dis 2018; 18: 538.

57 Menard S, Tchoufack JN, Maffo CN, et al. Insight into k13-propeller gene polymorphism and ex vivo DHA-response profiles from Cameroonian isolates. Malar J 2016; 15: 572

58 Hawkes M, Conroy AL, Opoka RO, et al. Slow clearance of Plasmodium falciparum in severe paediatric malaria, Uganda, 2011-2013. Emerg Infect Dis 2015; 21: 1237-39.

59 Miotto O, Amato R, Ashley EA, et al. Genetic architecture of artemisinin-resistant Plasmodium falciparum. Nat Genet 2015; 47: 226-34.

60 Mita T, Culleton R, Takahashi N, et al. Little polymorphism at the K13 propeller locus in worldwide Plasmodium falciparum populations prior to the introduction of artemisinin combination therapies. Antimicrob Agents Chemother 2016; 60: 3340-47.

61 Muwanguzi J, Henriques G, Sawa P, Bousema T, Sutherland CJ, Beshir KB. Lack of K13 mutations in Plasmodium falciparum persisting after artemisinin combination therapy treatment of Kenyan children. Malar J 2016; 15: 36.

62 Mvumbi DM, Bobanga TL, Kayembe J-MN, et al. Molecular surveillance of Plasmodium falciparum resistance to artemisininbased combination therapies in the Democratic Republic of Congo. PLoS One 2017; 12: e0179142.

63 Oboh MA, Ndiaye D, Antony HA, et al. Status of artemisinin resistance in malaria parasite Plasmodium falciparum from molecular analyses of the Kelch13 gene in southwestern Nigeria. Biomed Res Int 2018; 2018: 2305062.
64 Ocan M, Bwanga F, Okeng A, et al. Prevalence of K13-propeller gene polymorphisms among Plasmodium falciparum parasites isolated from adult symptomatic patients in northern Uganda. BMC Infect Dis 2016; 16: 428.

65 Ouattara A, Kone A, Adams M, et al. Polymorphisms in the K13-propeller gene in artemisinin-susceptible Plasmodium falciparum parasites from Bougoula-Hameau and Bandiagara, Mali. Am J Trop Med Hyg 2015; 92: 1202-06.

66 Tumwebaze P, Tukwasibwe S, Taylor A, et al. Changing antimalarial drug resistance patterns identified by surveillance at three sites in Uganda. J Iinfect Dis 2017; 215: 631-35.

67 Plucinski MM, Talundzic E, Morton L, et al. Efficacy of artemether-lumefantrine and dihydroartemisinin-piperaquine for treatment of uncomplicated malaria in children in Zaire and Uíge Provinces, Angola. Antimicrob Agents Chemother 2015; 59: 437-43.

68 Somé AF, Sorgho H, Zongo I, et al. Polymorphisms in K13, pfcrt, pfmdr1, pfdhfr, and pfdhps in parasites isolated from symptomatic malaria patients in Burkina Faso. Parasite 2016; 23: 60.

69 Tacoli C, Gai PP, Bayingana C, et al. Artemisinin resistanceassociated K13 polymorphisms of Plasmodium falciparum in southern Rwanda, 2010-2015. Am J Trop Med Hyg 2016; 95: 1090-93.

70 Talundzic E, Ndiaye YD, Deme AB, et al. Molecular epidemiology of Plasmodium falciparum kelch13 mutations in Senegal determined by using targeted amplicon deep sequencing. Antimicrob Agents Chemother 2017; 61: e02116-16.

71 Tawe L, Menegon M, Ramatlho P, et al. Molecular surveillance of Plasmodium falciparum drug resistance markers in clinical samples from Botswana. Am J Trop Med Hyg 2018; 99: 1499-503.

72 Torrentino-Madamet M, Fall B, Benoit N, et al. Limited polymorphisms in $k 13$ gene in Plasmodium falciparum isolates from Dakar, Senegal in 2012-2013. Malar J 2014; 13: 472.

73 Torrentino-Madamet M, Collet L, Lepère JF, et al. K13-propeller polymorphisms in Plasmodium falciparum isolates from patients in Mayotte in 2013 and 2014. Antimicrob Agents Chemother 2015; 59: 7878-81.

74 Madamet M, Kounta MB, Wade KA, et al. Absence of association between polymorphisms in the K13 gene and the presence of Plasmodium falciparum parasites at day 3 after treatment with artemisinin derivatives in Senegal. Int J Antimicrob Agents 2017; 49: 754-56.

75 Voumbo-Matoumona DF, Akiana J, Madamet M, Kouna LC, Lekana-Douki JB, Pradines B. High prevalence of Plasmodium falciparum antimalarial drug resistance markers in isolates from asymptomatic patients from the Republic of the Congo between 2010 and 2015. J Glob Antimicrob Resist 2018; 14: 277-83.

76 Voumbo-Matoumona DF, Kouna LC, Madamet M, Maghendji-Nzondo S, Pradines B, Lekana-Douki JB. Prevalence of Plasmodium falciparum antimalarial drug resistance genes in southeastern Gabon from 2011 to 2014. Infect Drug Resist 2018; 11: $1329-38$.

77 Taylor SM, Parobek CM, DeConti DK, et al. Absence of putative artemisinin resistance mutations among Plasmodium falciparum in sub-Saharan Africa: a molecular epidemiologic study. J Infect Dis 2014; 211: 680-88.

78 Dorkenoo AM, Yehadji D, Agbo YM, et al. Therapeutic efficacy trial of artemisinin-based combination therapy for the treatment of uncomplicated malaria and investigation of mutations in $k 13$ propeller domain in Togo, 2012-2013. Malar J 2016; 15: 331.

79 MalariaGEN Plasmodium falciparum Community Project. Genomic epidemiology of artemisinin resistant malaria. eLife 2016; 5: e08714.

80 Escalante AA, Lal AA, Ayala FJ. Genetic polymorphism and natura selection in the malaria parasite Plasmodium falciparum. Genetics 1998; 149: 189-202.

81 Miotto O, Almagro-Garcia J, Manske M, et al. Multiple populations of artemisinin-resistant Plasmodium falciparum in Cambodia. Nat Genet 2013; 45: 648-55.

82 Witkowski B, Lelièvre J, Barragán MJL, et al. Increased tolerance to artemisinin in Plasmodium falciparum is mediated by a quiescence mechanism. Antimicrob Agents Chemother 2010; 54: 1872-77.

83 Li G-Q, Guo X-B, Fu L-C, Jian H-X, Wang X-H. Clinical trials of artemisinin and its derivatives in the treatment of malaria in China. Trans R Soc Trop Med Hyg 1994; 88: S5-6. 
84 Bonnington CA, Phyo AP, Ashley EA, et al. Plasmodium falciparum Kelch 13 mutations and treatment response in patients in Hpa-Pun District, northern Kayin State, Myanmar. Malar J 2017; 16: 1-7.

85 Cheeseman IH, McDew-White M, Phyo AP, Sriprawat K, Nosten F, Anderson TJ. Pooled sequencing and rare variant association tests for identifying the determinants of emerging drug resistance in malaria parasites. Mol Biol Evol 2015; 32: 1080-90.

86 Wang J, Huang Y, Zhao Y, Ye R, Zhang D, Pan W. Introduction of F446I mutation in the K13 propeller gene leads to increased ring survival rates in Plasmodium falciparum isolates. Malar J 2018; 17: 248.

87 WWARN K13 Genotype-Phenotype Study Group. Association of mutations in the Plasmodium falciparum Kelch13 gene (Pf3D7_1343700) with parasite clearance rates after artemisinin-based treatments-a WWARN individual patient data meta-analysis. BMC Med 2019; 17: 1

88 Wang Z, Shrestha S, Li X, et al. Prevalence of K13-propeller polymorphisms in Plasmodium falciparum from China-Myanmar border in 2007-2012. Malar J 2015; 14: 168.

89 Anderson T, Nair S, White M, et al. Why are there so many independent origins of artemisinin resistance in malaria parasites? bioRxiv 2016; published online May 31. https://www.biorxiv.org/ content/10.1101/056291v1.full (preprint).

90 Singh GP, Goel P, Sharma A. Structural mapping of Kelch13 mutations associated with artemisinin resistance in malaria. J Struct Funct Genomics 2016; 17: 51-56.

91 Tsombeng FF, Gendrot M, Robert MG, Madamet M, Pradines B. Are $k 13$ and plasmepsin II genes, involved in Plasmodium falciparum resistance to artemisinin derivatives and piperaquine in southeast Asia, reliable to monitor resistance surveillance in Africa? Malar J 2019; 18: 285 .
92 Demas AR, Sharma AI, Wong W, et al. Mutations in Plasmodium falciparum actin-binding protein coronin confer reduced artemisinin susceptibility. Proc Natl Acad Sci USA 2018; 115: 12799-804.

93 Velavan TP, Nderu D, Agbenyega T, Ntoumi F, Kremsner PG. An alternative dogma on reduced artemisinin susceptibility: a new shadow from east to west. Proc Natl Acad Sci USA 2019; 116: 12611-12.

94 Henrici RC, Sutherland CJ. Alternative pathway to reduced artemisinin susceptibility in Plasmodium falciparum. Proc Natl Acad Sci USA 2018; 115: 12556-58.

95 Sharma AI, Demas AR, Hartl DL, Wirth DF. Reply to Velavan et al: polymorphisms of pfcoronin in natural populations: implications for functional significance. Proc Natl Acad Sci USA 2019; 116: 12613-14.

$96 \mathrm{Lu} \mathrm{F}$, Culleton R, Zhang M, et al. Emergence of indigenous artemisinin-resistant Plasmodium falciparum in Africa. $N$ Engl J Med 2017; 376: 991-93.

97 Yang C, Zhang H, Zhou R, et al. Polymorphisms of Plasmodium falciparum k13-propeller gene among migrant workers returning to Henan Province, China from Africa. BMC Infect Dis 2017; 17: 560.

(C) 2020 Elsevier Ltd. All rights reserved. 